\title{
Influência da extensão da palavra e local da ruptura na sílaba na fala de adolescentes e adultos gagos e fluentes
}

\author{
The influence of word extension and the position of speech \\ disruption within the syllable structure in the speech of \\ stuttering and fluent adolescents and adults
}

\author{
Fabiola Staróbole Juste ${ }^{1}$, Claudia Regina Furquim de Andrade ${ }^{2}$
}

\begin{abstract}
RESUMO
Objetivo: Verificar a influência da extensão da palavra e da posição dentro da sílaba na ocorrência de rupturas de fala em adolescentes e adultos gagos e fluentes. Métodos: Participaram do estudo 120 indivíduos, de ambos os gêneros, com idades entre 12 e 49 anos e 11 meses, divididos em quatro grupos: Grupo de Pesquisa 1 (GP1) - 30 adolescentes gagos; Grupo de Pesquisa 2 (GP2) - 30 adultos gagos; Grupo Controle 1 (GC1) - 30 adolescentes fluentes; Grupo Controle 2 (GC2) - 30 adultos fluentes. Foram coletadas amostras de fala contendo 200 sílabas fluentes. Nas amostras de fala, as palavras rompidas foram analisadas quanto à sua extensão (monossílaba, dissílaba, trissílaba e polissílaba) e quanto ao local da ruptura na sílaba (posição de ataque, núcleo ou coda). Resultados: Quanto à extensão, os grupos de falantes gagos não apresentaram predominância de rupturas em nenhuma das possibilidades, já para os falantes fluentes, as rupturas foram mais frequentes nas palavras monossilábicas. Em relação ao local da ruptura na sílaba, os falantes gagos apresentaram ruptura principalmente na posição de ataque, enquanto os fluentes apresentaram mais rupturas em posição de núcleo. Conclusão: De maneira geral, esses resultados sugerem que as rupturas de fala ocorrem no início das palavras e sílabas, indicando uma dificuldade na temporalização entre as velocidades de seleção e ativação fonológica, que ocorre independentemente da extensão da palavra.
\end{abstract}

Descritores: Distúrbios da fala; Gagueira; Métodos; Adolescentes; Adultos

\section{INTRODUÇÃO}

Entre os diversos aspectos linguísticos investigados na gagueira, a extensão da palavra rompida tem sido um tema frequentemente pesquisado, apresentando resultados divergentes.

As rupturas em palavras de maior extensão podem ocorrer pelo fato destas palavras necessitarem de mais tempo maior para serem programadas. Além disso, levando-se em conta uma situação de comunicação entre as pessoas, onde geralmente o indivíduo é submetido à pressão de "falar mais rápido", as

Trabalho realizado no Laboratório de Investigação Fonoaudiológica da Fluência, Funções da Face e Disfagia (LIF-FFFD) da Faculdade de Medicina, Universidade de São Paulo - USP - São Paulo (SP), Brasil.

(1) Departamento de Fisioterapia, Fonoaudiologia e Terapia Ocupacional da Faculdade de Medicina, Universidade de São Paulo - USP - São Paulo (SP), Brasil.

(2) Departamento de Fisioterapia, Fonoaudiologia e Terapia Ocupacional da Faculdade de Medicina, Universidade de São Paulo - USP - São Paulo (SP), Brasil.

Endereço para correspondência: Fabiola Staróbole Juste. R. Cipotânea, 51, Cidade Universitária, São Paulo (SP), Brasil, CEP: 05360-160. E-mail: fjuste@usp.br

Recebido em: 7/5/2010; Aceito em: 21/6/2010 palavras de maior extensão estariam mais sujeitas a serem gaguejadas $^{(1)}$.

A literatura ${ }^{(2)}$ aponta que as palavras de maior extensão apresentam uma maior complexidade articulatória, o que pode facilitar a ocorrência de rupturas.

$\mathrm{Um}_{\text {estudo }}{ }^{(3)}$ realizado com 19 adultos gagos falantes do Português Brasileiro teve como objetivo verificar a frequência da gagueira segundo a extensão da palavra. Os resultados deste estudo apontam que, das amostras pesquisadas, a maioria das palavras eram monossílabas, seguidas por dissílabas, trissílabas e polissílabas. Em relação às rupturas, a autora mostra que proporcionalmente a ordem se inverte, as palavras polissílabas foram as que apresentaram maior número de rupturas, seguidas pelas trissílabas, dissílabas, sendo as monossílabas as palavras menos gaguejadas.

Outro estudo ${ }^{(4)}$ realizado com crianças gagas e fluentes verificou seu desempenho de fala em não-palavras. Dentre os aspectos pesquisados, as autoras relatam que não houve variação na ocorrência de rupturas relacionada à extensão das não-palavras.

Em um estudo realizado com 32 gagos falantes do Alemão (15 adultos e 17 crianças) ${ }^{(5)}$, os autores investigaram uma série 
de fatores linguísticos que podem influenciar na gagueira, entre eles, a extensão da palavra. Os resultados indicaram que, analisando os dados isoladamente, tanto para os adultos quanto para as crianças, as rupturas de fala foram mais frequentes nas palavras de maior extensão.

Outro aspecto bastante discutido na literatura sobre a fala de indivíduos que gaguejam é a localização da ruptura dentro da palavra, onde se observa que as rupturas ocorrem com maior frequência na sílaba inicial ${ }^{(6-14)}$. Entretanto, essa análise quanto à posição da ruptura na palavra não contempla os monossílabos, que são palavras de grande frequência na Língua Portuguesa ${ }^{(15)}$. Alem disso, muito se discute sobre o local da ruptura na palavra, mas pouco foi pesquisado quanto ao local da ruptura dentro da sílaba.

Para o Português Brasileiro(16), a constituição básica da sílaba é a conhecida como CV (consoante-vogal). Além dessa, outras estruturas podem ser encontradas: V (vogal), CCV (consoante-consoante-vogal), CVC (consoante-vogalconsoante) e CVV (consoante-vogal-vogal).

A sílaba, entre outras atribuições, funciona como o principal elemento da estrutura prosódica, organizando hierarquicamente as unidades prosódicas em níveis mais altos (o "pé" e a palavra fonológica). A sílaba, sendo definida como uma categoria prosódica, organiza segmentos em sequência de acordo com seus valores de sonoridade ${ }^{(17)}$.

Cada sílaba possui um pico de sonoridade (núcleo) que geralmente é uma vogal, podendo ser rodeada de ambos os lados por segmentos de menor sonoridade, geralmente consoantes (ataque e coda) ${ }^{(18)}$, como por exemplo na palavra completo:

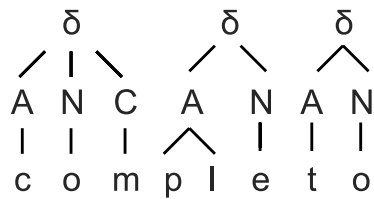

Essa estrutura plana define a sílaba como uma organização tripartida, constituída por onset (ataque - A), núcleo (N) e coda (C), sendo que desses elementos, o único universalmente obrigatório é o núcleo. As consoantes ficam nas posições de ataque e coda e as vogais na posição de núcleo.

Segundo esse autor ${ }^{(18)}$, esse modelo plano simples pode ser usado para explicar importantes observações em diferentes línguas. As línguas diferem quanto à estrutura da sílaba em relação aos seus elementos (ataque, núcleo e coda). Existem línguas que não permitem coda, outras línguas, permitem tanto ataque, núcleo e coda compostos (ex. dos ditongos e tritongos no núcleo para o Português).

Em 2003, um estudo ${ }^{(5)}$ comparou os achados linguísticos relacionados à gagueira em falantes do Inglês e do Alemão. De acordo com os autores, os achados dessas pesquisas devem ser comparados com muito cuidado, por se tratarem de línguas (Inglês e Alemão) de origens diversas, consequentemente, apresentando estruturas e regras diferentes. Ainda segundo os autores, uma dessas diferenças seria em relação à sílaba, mais especificamente em relação ao ataque.

Para o Alemão, a posição de ataque na sílaba é obrigatória, portanto, em palavras iniciadas por vogal, um golpe glotal é inserido antes da vogal inicial da palavra, funcionando com o ataque da sílaba. Portanto, toda a palavra iniciada por vogal no Alemão é precedida pelo ataque glotal, o que aumenta a tensão muscular para a sua produção, podendo aumentar o risco de ocorrência de rupturas, explicando o achado de que no Alemão ocorrem mais rupturas em palavras iniciadas por vogais quando comparado ao Inglês. Esta comparação também pode ser feita em relação ao Português, que da mesma forma que o Inglês, permite que uma sílaba seja iniciada pelo núcleo da sílaba.

A partir da literatura apresentada os objetivos propostos pelo presente estudo são verificar se a extensão da palavra influencia a ocorrência de rupturas e verificar a existência de um padrão de localização da ruptura dentro da sílaba na fala de adolescentes e adultos gagos e fluentes.

\section{MÉTODOS}

Os processos de seleção e avaliação seguiram os processos éticos pertinentes: parecer da Comissão de Ética (CAPPesq HCFMUSP $n^{\circ}$ 0823/07) e a assinatura do Termo de Consentimento Livre e Esclarecido, pelos responsáveis dos participantes.

\section{Participantes}

Participaram desta pesquisa 120 indivíduos gagos e fluentes, pareados por gênero e idade, residentes no município de São Paulo e Grande São Paulo, que concordaram, através de assinatura do termo de consentimento, na realização dos procedimentos propostos nesta pesquisa.

Os participantes desse estudo foram divididos em quatros grupos:

a) grupo de pesquisa 1 (GP1): foi composto por 30 adolescentes, com idades entre 12 anos a 17 anos e 11 meses, 19 do gênero masculino e 11 do gênero feminino, com diagnóstico de gagueira, sem qualquer outro déficit comunicativo, neurológico e cognitivo associado;

b) grupo de pesquisa 2 (GP2): foi composto por 30 adultos, com idades entre 18 anos a 49 anos e 11 meses, 24 do gênero masculino e seis do gênero feminino, com diagnóstico de gagueira, sem qualquer outro déficit comunicativo, neurológico e cognitivo associado;

c) grupo controle 1 (GC1); foi composto por 30 adolescentes, pareados ao GP1 quanto ao gênero e idade, sem queixa de gagueira ou qualquer déficit comunicativo, neurológico e cognitivo, associado;

d) grupo controle 2 (GC2): foi composto por 30 adultos, pareados ao GP2 quanto ao gênero e idade, sem queixa de gagueira ou qualquer déficit comunicativo, neurológico e cognitivo, associado.

Os critérios de inclusão dos participantes do GP1 e GP1 foram:

a) apresentar pontuação do perfil da fluência fora dos valores de referência para a idade ${ }^{(19)}$;

b) receber 11 pontos ou mais para os adolescentes e 18 pontos ou mais para os adultos (gravidade equivalente a pelo menos "leve") no Stuttering Severity Instrument - 3 $\left(\mathrm{SSI}-3^{(20)}\right)$. 

foram:

Os critérios de inclusão dos participantes de GC1 e GC2

a) apresentar pontuação do perfil da fluência de acordo com os valores de referência para a idade ${ }^{(19)}$;

b) receber um total de dez pontos para adolescentes ou 17 pontos para adultos (gravidade equivalente a "muito leve") ou menos no SSI-3 ${ }^{(20)}$.

\section{Material}

Para registro e análise das amostras de fala, foram utilizados filmadora Sony® Digital DCR-SR85 fixada em tripé, microcomputador Dell® Studio Hybrid, e netbook HP mini 1120BR.

As amostras de fala foram coletadas e analisadas segundo o Protocolo do Perfil da Fluência ${ }^{(21)}$ e pelo Stuttering Severity Instrument - SSI-3 ${ }^{(20)}$ para determinar a gravidade de gagueira para GP1 e GP2.

\section{Procedimento}

\section{Coleta das amostras de fala}

Para a obtenção das amostras de fala, adotou-se uma metodologia ${ }^{(21)}$ onde foi apresentada uma figura aos participantes e dada a seguinte ordem: "por favor olhe essa figura e me fale tudo o que você quiser sobre ela". O discurso só foi interrompido por perguntas e/ou comentários, nos casos em que houve a necessidade de incentivar a produção do discurso para a obtenção de 200 sílabas fluentes (número de sílabas fluentes necessárias para a análise da amostra). Quanto ao tempo previsto para cada coleta, foi estabelecido o mínimo de cinco minutos.

\section{Análise das amostras de fala}

Após a coleta das amostras de fala dos participantes, as mesmas foram transcritas e caracterizadas segundo as tipologias das rupturas, extensão das palavras rompidas e posição da ruptura na sílaba.

Em razão da grande variabilidade do número de informações e resultados gerados nas análises citadas acima, foi considerada necessária uma análise de confiabilidade. Para a avaliação da fidedignidade das análises realizadas, foi utilizada a validação dos resultados por compatibilização interjuízes.

Para a análise dos resultados, foram selecionadas aleatoriamente cinco amostras de fala e submetidas a uma re-análise por dois juízes Fonoaudiólogos com ampla experiência na área de gagueira. Os juízes receberam a instrução de assistir as gravações em vídeo e analisar as amostras de fala de acordo com os critérios de análise estabelecidos nessa pesquisa.
Durante a análise das amostras de fala pelos juízes, não houve contato dos mesmos com o pesquisador. Após essa análise, obteve-se um índice de concordância de $96 \%$ para o juiz 1 e $92 \%$ para o juiz 2.

\section{- Caracterização da tipologia das rupturas}

As rupturas foram classificadas e analisadas segundo o Protocolo do Perfil da Fluência ${ }^{(21)}$ divididas em:

- rupturas comuns - hesitações,interjeições, revisões, palavras não terminadas, repetições de palavras, segmentos e frases;

- rupturas gagas - repetições de sons e sílabas, prolongamentos, bloqueios, pausas e intrusões.

\section{- Caracterização da extensão da palavra rompida}

As rupturas foram analisadas quanto à sua extensão e divididas em palavras monossílabas, dissílabas, trissílabas e polissílabas.

A análise da extensão da palavra rompida não considerou o número total de rupturas dos grupos. As rupturas descartadas para essa análise foram hesitação, palavra incompleta, pausa e intrusão. Para a tipologia de repetição de segmentos, foram analisadas todas as palavras do evento (ex: Na sentença "u mininu u mininu foi nu parqui", "u" foi considerado monossílabo e "mininu" trissílabo, tendo duas classificações para um único evento).

\section{- Caracterização da posição da ruptura na sílaba}

Esta é uma análise intra-sílaba, portanto, somente as rupturas que ocorreram em fonemas isolados foram analisadas. Essa análise foi restrita às rupturas do tipo repetição de sílabas (apenas para as sílabas tipo "V", ou seja, compostas apenas por uma vogal - ex. água), repetição de som, prolongamento e bloqueio.

Para cada evento, foi analisado em que local da sílaba ocorreu a ruptura (ataque, núcleo ou coda). Para essa análise, a estrutura silábica utilizada foi a tenária ${ }^{(18)}$ (estrutura baseada apenas na divisão entre ataque núcleo e coda sem subdivisões).

\section{Análise estatística}

Para a análise estatística dos dados foi utilizado o teste análise de variância (ANOVA), com nível de significância de 5\%.

\section{RESULTADOS}

As tabelas abaixo mostram os resultados obtidos de acordo com as análises propostas no Método.

$\mathrm{Na}$ Tabela 1 observa-se que as rupturas de fala foram mais frequentes nas palavras monossílabas, sendo essa pre-

Tabela 1. Análise intra-grupos de adolescentes (GP 1 e GC 1) e adultos (GP 2 e GC 2) quanto à extensão das palavras rompidas

\begin{tabular}{|c|c|c|c|c|c|c|c|c|c|c|c|c|}
\hline \multirow{2}{*}{ Grupos } & \multicolumn{3}{|c|}{ Monossílabas } & \multicolumn{3}{|c|}{ Dissílabas } & \multicolumn{3}{|c|}{ Trissílabas } & \multicolumn{3}{|c|}{ Polissílabas } \\
\hline & Total & Média & DP & Total & Média & DP & Total & Média & $\mathrm{DP}$ & Total & Média & DP \\
\hline GP 1 & 368 & 12,26 & 6,22 & 244 & 8,13 & 5,99 & 116 & 3,86 & 3,14 & 31 & 1,03 & 1,47 \\
\hline GC 1 & 136 & 4,53 & 2,38 & 44 & 1,46 & 1,54 & 4 & 0,13 & 0,34 & 0 & 0 & 0 \\
\hline GP 2 & 429 & 10,96 & 5,63 & 331 & 11,03 & 7,46 & 121 & 4,03 & 2,07 & 38 & 1,3 & 0,98 \\
\hline $\mathrm{GC} 2$ & 123 & 4,1 & 3,05 & 40 & 1,56 & 1,22 & 13 & 0,43 & 0,72 & 2 & 0,06 & 0,25 \\
\hline
\end{tabular}

Legenda: DP = desvio-padrão 
dominância significativa para todos os grupos testados, GP1 $[\mathrm{F}(3,116)=33,45 \mathrm{p}<0,001] ; \mathrm{GC} 1[\mathrm{~F}(3,116)=64,79 \mathrm{p}<0,001]$; GP2 $[\mathrm{F}(3,116)=15,40 \mathrm{p}<0,001] ; \mathrm{GC} 2[\mathrm{~F}(3,116)=25,02$ $\mathrm{p}<0,001]$.

A Tabela 2 mostra a compatibilização entre o número de palavras rompidas segundo sua extensão e o número de palavras da amostra para cada caso. Em relação aos grupos de falantes gagos, os resultados percentuais apontam haver uma distribuição uniforme das rupturas em relação à extensão, os seja, quando comparadas com o número total de palavras da amostra, a maior ocorrência de rupturas em palavras monossílabas é neutralizada. Quanto aos grupos fluentes, observa-se que, mesmo sendo as palavras mais frequentes das amostras, as palavras monossílabas foram realmente as que sofreram mais rupturas, tanto para GC1 quanto para GC2.

Tabela 2. Compatibilização entre o número de palavras rompidas quanto a sua extensão em relação ao número total de palavras das amostras para os grupos de adolescentes (GP 1 e GC 1) e adultos (GP 2 e GC 2)

\begin{tabular}{lcccc}
\hline Grupo & Monossílabas & Dissílabas & Trissílabas & Polissílabas \\
\hline \multirow{2}{*}{ GP 1 } & $368 / 1674$ & $244 / 1199$ & $116 / 462$ & $31 / 135$ \\
& $21,9 \%$ & $20,3 \%$ & $25,1 \%$ & $22,9 \%$ \\
\hline \multirow{2}{*}{ GC 1} & $136 / 1661$ & $44 / 1207$ & $4 / 463$ & $0 / 130$ \\
& $8,1 \%$ & $3,6 \%$ & $0,86 \%$ & - \\
\hline \multirow{2}{*}{ GP 2 } & $429 / 1671$ & $331 / 1196$ & $121 / 454$ & $38 / 139$ \\
& $25,6 \%$ & $27,6 \%$ & $26,6 \%$ & $27,3 \%$ \\
\hline \multirow{2}{*}{ GC 2 } & $123 / 1655$ & $40 / 1198$ & $13 / 461$ & $2 / 120$ \\
& $7,4 \%$ & $3,3 \%$ & $2,8 \%$ & $1,6 \%$ \\
\hline
\end{tabular}

Na Tabela 3 observa-se que para os grupos de falantes gagos, as rupturas foram mais frequentes na posição de ataque da sílaba, com diferenças significativas para adolescentes GP1 $[\mathrm{F}(2,88)=9,70 \mathrm{p}=0,002]$ e para adultos $\mathrm{GP} 2[\mathrm{~F}(2,88)=19,76$ $\mathrm{p}<0,001]$ quando comparados aos valores de rupturas nas posições de núcleo e coda. Em relação aos grupos fluentes, as rupturas foram mais frequentes na posição de núcleo das sílabas. Para GC1, a comparação foi realizada entre rupturas em núcleo e coda $[\mathrm{F}(1,58)=18,88 \mathrm{p}<0,001]$, já que não foram observadas ocorrência de rupturas em posição de ataque para este grupo. Em relação à GC2, não foi realizado cálculo estatístico, já que não foram observadas ocorrências de rupturas em posição de ataque e coda, este grupo apresentou somente rupturas em posição de núcleo das sílabas.

A Tabela 4 detalha as rupturas ocorridas em posição de ataque quanto à sua tipologia. Observa-se que para os grupos de falantes gagos, o bloqueio foi a tipologia predominante na posição de ataque das sílabas, diferenciando-se estatisticamente das outras tipologias - GP1 $[\mathrm{F}(2,87)=19,32 \mathrm{p}<0,001] \mathrm{e}$ GP2 $[\mathrm{F}(2,87)=14,94 \mathrm{p}<0,001]$. Como apontado na Tabela 3 , não foram observadas rupturas em posição de ataque na sílaba para os grupos de falantes fluentes.

$\mathrm{Na}$ Tabela 5 são apresentadas as tipologias de rupturas em posição de núcleo das sílabas. Para os grupos gagos, o prolongamento foi a ruptura mais frequente na posição de núcleo diferenciando-se estatisticamente das demais tipologias $\mathrm{GP} 1[\mathrm{~F}(2,87)=9,57 \mathrm{p}<0,001]$ e GP2 $[\mathrm{F}(2,87)=4,45 \mathrm{p}=0,002]$. Nos grupos fluentes, as rupturas em posição de núcleo foram exclusivamente prolongamentos.

Como apontado na Tabela 3, as rupturas em posição de coda foram pouco frequentes quando comparadas às rupturas em outras posições. Para todos os grupos testados, o prolongamento foi a única tipologia encontrada nas rupturas em posição de coda.

\section{DISCUSSÃO}

A literatura aponta que as rupturas são mais frequentes em palavras mais extensas devido à sua maior complexidade articulatória e a necessidade de mais tempo para serem programadas $^{(1,2)}$. Entretanto, os resultados do presente estudo indicaram que a extensão das palavras não foi um fator que influenciou a ocorrência de rupturas na fala de adolescentes e

Tabela 3. Comparação intra-grupos da distribuição das rupturas de acordo com sua posição na sílaba

\begin{tabular}{lccccccccc}
\hline Grupo & \multicolumn{3}{c}{ Ataque } & \multicolumn{3}{c}{ Núcleo } & \multicolumn{3}{c}{ Coda } \\
\cline { 2 - 10 } & Total & Média & DP & Total & Média & DP & Total & Média & DP \\
\hline GP 1 & 218 & 7,26 & 5,40 & 118 & 3,93 & 2,25 & 10 & 0,33 & 0,71 \\
GC 1 & 0 & 0 & 0 & 36 & 1,2 & 1,32 & 3 & 0,1 & 0,30 \\
GP 2 & 446 & 14,86 & 14,68 & 164 & 6,46 & 6,47 & 8 & 0,26 & 0,52 \\
GC 2 & 0 & 0 & 0 & 43 & 1,43 & 1,25 & 0 & 0 & 0 \\
\hline
\end{tabular}

Legenda: DP = desvio-padrão

Tabela 4. Comparação intra-grupos da distribuição das rupturas por tipologia em posição de ataque na sílaba

\begin{tabular}{lccccccccc}
\hline Grupo & \multicolumn{3}{c}{ Bloqueio } & \multicolumn{2}{c}{ Repetição de som/sílaba (V) } & \multicolumn{3}{c}{ Prolongamento } \\
\cline { 2 - 9 } & Total & Média & DP & Total & Média & DP & Total & Média & DP \\
\hline GP 1 & 143 & 4,76 & 4,88 & 30 & 1 & 2,55 & 12 & 0,4 & 0,56 \\
GC 1 & 0 & 0 & 0 & 0 & 0 & 0 & 0 & 0 & 0 \\
GP 2 & 359 & 11,96 & 14,40 & 62 & 2,06 & 2,61 & 25 & 0,83 & 3,14 \\
GC 2 & 0 & 0 & 0 & 0 & 0 & 0 & 0 & 0 \\
\hline
\end{tabular}

Legenda: DP = desvio-padrão 
Tabela 5. Comparação intra-grupos da distribuição das rupturas por tipologia em posição de núcleo na sílaba

\begin{tabular}{lccccccccc}
\hline Grupo & \multicolumn{3}{c}{ Bloqueio } & \multicolumn{3}{c}{ Repetição de som/sílaba (V) } & \multicolumn{2}{c}{ Prolongamento } \\
\cline { 2 - 9 } & Total & Média & DP & Total & Média & DP & Total & Média & DP \\
\hline GP 1 & 26 & 0,86 & 1,38 & 33 & 1,1 & 1,39 & 92 & 3,6 & 3,13 \\
GC 1 & 0 & 0 & 0 & 0 & 0 & 0 & 36 & 1,2 & 1,32 \\
GP 2 & 41 & 1,36 & 1,88 & 37 & 1,23 & 1,65 & 116 & 3,86 & 6,17 \\
GC 2 & 0 & 0 & 0 & 0 & 0 & 0 & 43 & 1,43 \\
\hline
\end{tabular}

Legenda: DP = desvio-padrão

adultos gagos, já que não foi observada predominância para nenhuma extensão especificamente. Outros fatores como a classe gramatical das palavras ${ }^{(22-27)}$ e local da ruptura na palavra ${ }^{(28)}$ parecem contribuir de maneira mais efetiva para a ocorrência de rupturas na fala de pessoas que gaguejam.

Ainda quanto à extensão das palavras rompidas, um dado interessante foi encontrado para os grupos de adolescentes e adultos fluentes. Para estes grupos, as rupturas de fala foram mais frequentes nas palavras monossílabas. Uma possível explicação para esse resultado é que, em estudos anteriormente realizados $^{(22-27)}$, verificou-se que para os falantes fluentes, as rupturas de fala ocorreram predominantemente nas palavras funcionais, sendo destas, o artigo, a preposição e os pronomes as classes gramaticais com maior número de ocorrências de rupturas. No Português Brasileiro, nos artigos (tanto diretos quanto os indiretos) e nas demais palavras funcionais (conjunções, preposições, pronomes e interjeições), pode-se observar uma grande ocorrência de palavras monossilábicas ${ }^{(15)}$.

Em relação aos resultados quanto a posição de rupturas dentro da sílaba, mais uma vez percebemos uma diferenciação entre os grupos gagos e fluentes.

Para os grupos de falantes gagos, as rupturas de fala foram mais frequentes nas sílabas iniciais ${ }^{(28)}$ e na posição de ataque da sílaba, sendo o bloqueio a tipologia predominante, resultado que está de acordo com os achados de outro estudo ${ }^{(27)}$. A maior ocorrência de bloqueios pode indicar que na posição de ataque da sílaba, a velocidade de seleção do fonema é maior do que sua velocidade de ativação ${ }^{(11)}$. As rupturas de fala ocorrem devido a uma assincronia entre a velocidade de seleção e ativação do fonema alvo. Dessa forma, quando a velocidade de seleção do fonema é maior do que a sua velocidade de ativação, o processamento será bloqueado. Caso a velocidade de seleção do fonema seja mais lenta do que sua velocidade de ativação, o processamento será prolongado ${ }^{(11)}$.

A ruptura, tanto na primeira sílaba da palavra quanto no primeiro fonema da sílaba, é fato conhecido na literatura como word-initial-effect ${ }^{(27,29)}$. Assim como a ruptura na sílaba inicial, a ruptura no primeiro fonema da sílaba parece ser decorrente de uma dificuldade em iniciar a produção da estrutura linguística ${ }^{(1,27)}$.

Em relação aos falantes fluentes, as rupturas foram mais frequentes nas sílabas finais ${ }^{(28)}$, na posição de núcleo das sílabas.

Quanto à posição de núcleo, ambos os grupos gagos e fluentes apresentaram predominância de prolongamentos, estando também esse resultado de acordo com outro estudo(27). A posição de núcleo é preenchida exclusivamente pelas vogais. Articulatoriamente, a diferença entre vogais e consoantes é que para as vogais, a passagem do ar é livre, não há nenhum articulador na cavidade oral que obstrua seu caminho, ao contrário das consoantes ${ }^{(16)}$. Essa característica pode ser uma possível explicação para o fato das vogais serem mais facilmente prolongadas do que bloqueadas.

As rupturas em posição de coda foram menos frequentes para ambos os grupos, o que pode ser explicado pelo fato de que a configuração silábica predominante para o Português é a sílaba $\mathrm{CV}^{(16)}$ contendo apenas ataque e núcleo. Os resultados apontaram ainda que para ambos os grupos, o prolongamento foi a única tipologia encontrada em posição de coda das sílabas. O prolongamento também foi a tipologia de maior frequência nas silabas finais das palavras, sugerindo que sua ocorrência em posição final (tanto de palavras quanto de sílabas) pode ser uma estratégia utilizada para facilitar a coarticulação entre as palavras, mantendo a fluência da emissão.

A literatura aponta que o processo de recuperação de um programa motor de uma unidade inicia-se pouco antes da expressão da unidade anterior. Dessa forma, quando o falante está articulando uma unidade, significa que já tem disponível o programa motor da unidade subsequente àquela que está sendo articulada. Para que a fluência seja alcançada, o mecanismo utilizado pelo falante é prolongar a parte final da palavra anterior, promovendo uma junção com a palavra seguinte ${ }^{(12)}$.

\section{CONCLUSÃO}

Os resultados indicaram que para os falantes gagos, a extensão das palavras não foi um fator que influenciou a ocorrência de rupturas de fala. Para os grupos fluentes, as rupturas de fala foram mais frequentes nas palavras monossílabas, fator correlacionado com a classe gramatical das palavras.

Em relação à posição da ruptura na sílaba, a posição de ataque foi a mais rompida para os grupos gagos e a posição de núcleo para os grupos fluentes.

De maneira geral, esses resultados sugerem que as rupturas de fala ocorrem no início das palavras e sílabas, indicando uma dificuldade na temporalização entre as velocidades de seleção e ativação fonológica, que ocorre independentemente da extensão da palavra.

\section{AGRADECIMENTOS}

Agradecemos à Fundação de Amparo à Pesquisa do Estado de São Paulo (FAPESP) pelo apoio concedido para a realização dessa pesquisa, sob processo número 07/03702-5, na forma de bolsa de Pós-Doutorado. 


\begin{abstract}
Purpose: To verify the influence of word extension and the position of speech disruption within the syllable structure in the speech of stuttering and fluent adolescents and adults. Methods: Participants were 120 individuals of both genders, with ages between 12 and 49 years and 11 months, who were divided into four groups: Research Group 1 (RG1) - 30 adolescents who stutter; Research Group 2 (RG2) - 30 adults who stutter; Control Group 1 (CG1) - 30 adolescents who do not stutter; Control Group 2 (CG2) - 30 adults who do not stutter. Speech samples containing 200 fluent syllables were gathered. Stuttered words were analyzed according to their extension (monosyllables, two, three or more syllables) and to the position of the disruption within the syllable (onset, nucleus or coda). Results: Speech disruptions did not prevail in any of the possible types of word extension for the stuttering groups. However, for the groups of fluent individuals, speech disruptions were more frequent on monosyllabic words. As for the analyses of speech disruptions within the syllable, stutterers presented speech disruptions mainly on the onset position, whereas fluent individuals presented speech disruptions mainly on the nucleus of the syllable. Conclusion: Overall, results suggest that speech disruptions occur at the beginning of words and syllables, thus indicating difficulty in synchronizing phonological selection and activation. This fact happens independently of word extension.
\end{abstract}

Keywords: Speech disorders; Stuttering; Methods; Adolescents; Adults

\title{
REFERÊNCIAS
}

1. van Lieshout PH, Hulstijn W, Peters HF. From planning to articulation in speech production: what differentiates a person who stutters from a person who does not stutter? J Speech Lang Hear Res. 1996;39(3):54664.

2. Bloodstein O. Incipient and developed stuttering as two distinct disorders: resolving a dilemma. J Fluency Disord. 2001;26(1):67-73.

3. Schiefer AM. Abordagem psicolinguística da fluência. In: Ferreira LP, Befi-Lopes DM, Limongi SCO. Tratado de fonoaudiologia. São Paulo: Rocca; 2004. cap. 83, p. 1035-9.

4. Hakim HB, Ratner NB. Nonword repetition abilities of children who stutter: an exploratory study. J Fluency Disord. 2004;29(3):179-99.

5. Dworzynski K, Howell P, Natke U. Predicting stuttering from linguistic factors for German speakers in two age groups. J Fluency Disord. 2003;28(2):95-112; quiz 112-3.

6. Wingate ME. Stutter events and linguistic stress. J Fluency Disord. 1984;9(4):295- 300.

7. Ratner NB. Language complexity and stuttering in children. Top Lang Disord. 1995;15(3):32-47.

8. Ratner NB. Performance or capacity, the model still requires definitions and boundaries it doesn't have: the demands and capacities model. J Fluency Disord. 2000;25(4):337-46.

9. Ambrose NG, Yairi E. Normative disfluency data for early childhood stuttering. J Speech Lang Hear Res. 1999;42(4):895-909. Comment in: J Speech Lang Hear Res. 2001;44(3):585-92. J Speech Lang Hear Res. 2001;44(3):593-7.

10. Hubbard CP. Stuttering, stressed syllables, and word onsets. J Speech Lang Hear Res. 1998;41(4):802-8.

11. Conture EG. Stuttering: its nature, diagnosis, and treatment. Boston: Allyn \& Bacon; 2000.

12. Pereira MMB. Análise linguística da gagueira. São Paulo: AM3; 2003.

13. Ambrose NG, Yairi E. The role of repetition units in the differential diagnosis of early childhood incipient stuttering. Am J Speech Lang Pathol. 1995;4(1):82-8.

14. Pellowski MW, Conture EG. Characteristics of speech disfluency and stuttering behaviors in 3- and 4-year-old children. J Speech Lang Hear Res. 2002;45(1):20-34.

15. Rosa MC. Classes de palavras, tipos de significado e questões relacionadas. In: Rosa MC. Introdução à morfologia. 3a ed.São Paulo: Contexto; 2003. p. 91-114.
16. Santos RS, Souza PC. Fonética. In: Fiorin JL, organizador. Introdução à linguística II: princípios de análise. 3a ed. São Paulo: Contexto; 2004. p. 9-31.

17. Selkirk EO. The syllable. In: van der Hutst H, Smith N, editors. The structure of phonological representations. USA: Foris Pubns; 1982, p. 337-383.

18. Blevins J. The syllable in phonological theory. In: Goldsmith JA, editor. The handbook of phonological theory. Cambridge, Mass., USA: Blackwell; c1996. cap. 06. p. 206-44.

19. Andrade CRF. Perfil da fluência da fala: parâmetro comparativo diferenciado por idade para crianças, adolescentes, adultos e idosos (CD-ROM). Barueri: Pró-Fono; 2006.

20. Riley GD. A stuttering severity instrument for children and adults. Austin: Pro-Ed; 1994.

21. Andrade CRF. Protocolo para avaliação da fluência da fala. Pró-Fono. 2000;12(2):131-4.

22. Juste F, Andrade CRF. Tipologia das rupturas de fala e classes gramaticais em crianças gagas e fluentes. Pró-Fono. 2006;18(2):129-40.

23. Au-Yeung J, Howell P, Pilgrim L. Phonological words and stuttering on function words. J Speech Lang Hear Res. 1998;41(5):1019-30.

24. Howell P, Au-Yeung J, Sackin S. Exchange of stuttering from function words to content words with age. J Speech Lang Hear Res. 1999;42(2):345-54.

25. Au-Yeung J, Gomez IV, Howell P. Exchange of disfluency with age from function words to content words in spanish speakers who stutter. J Speech Lang Hear Res. 2003;46(3):754-65.

26. Savage C, Howell P. Lexical priming of function words and content words with children who do, and do not, stutter. J Commun Disord. 2008;41(6):459-84.

27. Natke U, Sandrieser P, van Ark M, Pietrowsky R, Kalveram KT. Linguistic stress, within-word position, and grammatical class in relation to early childhood stuttering. J Fluency Disord. 2004;29(2):109-22.

28. Juste FS, Andrade CRF. Influência da tonicidade e local da ruptura na palavra em adolescentes e adultos gagos e fluentes. Pró-Fono. 2010;22(3):175-82.

29. Natke U, Grosser J, Sandrieser P, Kalveram KT. The duration component of the stress effect in stuttering. J Fluency Disord. 2002;27(4):305-17; quiz 318. 